\title{
A note on Friedmann equation of FRW universe in deformed Hořava-Lifshitz gravity from entropic force
}

\author{
Shao-Wen Wei*, Yu-Xiao Liu ${ }^{\dagger}$, Yong-Qiang Wang ${ }^{\ddagger}$ \\ Institute of Theoretical Physics, Lanzhou University, Lanzhou 730000, P. R. China
}

\begin{abstract}
With entropic interpretation of gravity proposed by Verlinde, we obtain the Friedmann equation of the Friedmann-Robertson-Walker universe for the deformed Hořava-Lifshitz gravity. It is shown that, when the parameter of Hořava-Lifshitz gravity $\omega \rightarrow \infty$, the modified Friedmann equation will go back to the one in Einstein gravity. This results may imply that the entropic interpretation of gravity is effective for the deformed Hořava-Lifshitz gravity.

PACS numbers: 04.70.Dy
\end{abstract}

Keywords: Entropic force, Horava-Lifshitz gravity, Friedmann equation

\footnotetext{
${ }^{*}$ E-mail: weishaow06@lzu.edu.cn

$\dagger$ E-mail: liuyx@lzu.edu.cn, corresponding author

$\ddagger$ E-mail: yqwang@lzu.edu.cn
} 
Recently, motivated by Lifshitz theory in solid state physics [1], Hořava proposed a new gravity theory at a Lifshitz point [2 4]. The theory is usually referred to as the HořavaLifshitz (HL) theory. It has manifest 3-dimensional spatial general covariance and time reparametrization invariance. This is a non-relativistic renormalizable theory of gravity and recovers the four dimensional general covariance only in an infrared limit. Thus, it may be regarded as a UV complete candidate for general relativity.

The black hole solutions in this gravity theory have been attracted much attention. LüMei-Pope (LMP) first obtained the spherically symmetric black hole solution with dynamical parameter $\lambda$ in asymptotically Lifshitz spacetimes [5]. Following, other black hole solutions and cosmological solutions were obtained [ 6 - 19]. The thermodynamic properties and dynamical properties of different black hole solutions were also investigated intensively [20][45].

On the other hand, Verlinde [46] recently made a robust suggestion that gravity can be explained as an entropic force caused by the changes in the information associated with the positions of material bodies. This idea implies that gravity is not fundamental. In fact, the similar idea can be traced back to the work given by Sakharov about forty years ago [47]. In the original article, with the assumption of the entropic force and the Unruh temperature, Verlinde obtained the second law of Newton mechanics. With the holographic principle and the equipartition law of energy, Verlinde also derived the Newtonian law of gravitation. On the other side, using the equipartition law of energy for the horizon degrees of freedom together with the thermodynamic relation $S=E /(2 T)$, Padmanabhan also obtained the Newton's law of gravity [48].

With the idea of entropic force, some work has been carried out recently. At almost the same time, Shu and Gong [49], Cai, Cao and Ohta [50] obtained the Friedmann equations, respectively. Smomlin derived the Newtonian gravity in loop quantum gravity [51]. On the other hand, Makela pointed out that Verlinde's entropic force is actually the consequence of a specific microscopic model of spacetime [52]. Caravelli and Modesto also applied the similar ideas to the construction of holographic actions from black hole entropy [53]. In [54], $\mathrm{Li}$ and Wang showed that the holographic dark energy can be derived from the entropic force formula. Gao [55] gave a modified entropic force in the Debye's model. Wang [56] regarded the coulomb force as an entropic force. Zhang, Gong and Zhu [57] discussed three different corrections to the area law of entropy and obtained the modified Friedmann equations. 
Other applications can be seen in [58 60].

In this paper, we will study the Friedmann equation of the Friedmann-Robertson-Walker (FRW) in deformed HL gravity from the view of entropic force. Let us start with an (3 +1 -dimensional FRW universe, which is described by the metric

$$
d s^{2}=-d t^{2}+a^{2}(t)\left(\frac{d r^{2}}{1-k r^{2}}+r^{2} d \Omega_{2}^{2}\right),
$$

where $k$ denotes the spatial curvature constant with $k=+1,0$ and -1 corresponding to a closed, flat and open universe, respectively. $d \Omega_{2}^{2}$ is the line element of a two-dimensional unit sphere. Adopting a new coordinate $\tilde{r}=a(t) r$, the metric (1) will become

$$
d s^{2}=h_{a b} d x^{a} d x^{b}+\tilde{r}^{2} d \Omega_{2}^{2}
$$

with $x^{0}=t, x^{1}=r, h_{a b}=\operatorname{diag}\left(-1, a^{2} /\left(1-k r^{2}\right)\right)$. The dynamical apparent horizon $\tilde{r}_{A}$ is determined by $h^{a b} \partial_{a} \tilde{r} \partial_{b} \tilde{r}=0$, and is given by

$$
\tilde{r}_{A}=\frac{c}{\sqrt{H^{2}+k / a^{2}}}
$$

where $H \equiv \dot{a} / a$ is the Hubble parameter and $c$ is the speed of light. The overdot stands for the derivative with respect to the cosmic time $t$. Suppose that the energy-momentum tensor $T_{\mu \nu}$ of the matter in the universe has the form of a perfect fluid $T_{\mu \nu}=(\rho+p) U_{\mu} U_{\nu}+p g_{\mu \nu}$, where $U^{\mu}$ denotes the four-velocity of the fluid and $\rho$ and $p$ are the energy density and pressure, respectively. The energy conservation law $\nabla_{\mu} T^{\mu \nu}=0$ gives the continuity equation in the form

$$
\dot{\rho}+3 H(\rho+p)=0 .
$$

The time-component of the Einstein equation is nothing but the standard Friedmann equation

$$
H^{2}+\frac{k}{a^{2}}=\frac{8 \pi G}{3} \rho
$$

which describes the dynamical evolution of the universe model. It is proved in [61] that the Friedmann equation (5) can be derived by the first law of thermodynamics. Much work in this field has been made [62 67]. Assuming that the entropy/area relation in Hořava-Lifshitz gravity is $S=A / 4$, the standard Friedmann equation was obtained in [30] from the clausius relation. 
Next, we will devote our efforts to obtain the modified Friedmann equation in the deformed HL gravity from the entropic force. First, we assume that the temperature corresponding to the apparent horizon is

$$
T=\frac{\hbar c}{2 \pi k_{B} \tilde{r}_{A}} .
$$

The entropy $S$ depends on the gravity theory and takes different forms for different gravity theories. In the deformed HL gravity, it has the form

$$
S=\frac{k_{B} A c^{3}}{4 G \hbar}+\frac{k_{B} \pi}{\omega} \ln \frac{A c^{3}}{G \hbar} .
$$

This entropy/area relation has a logarithmic term, which is a characteristic of HL gravity theory. However, as the parameter $\omega \rightarrow \infty$, it will go back to the one in Einstein gravity. From the definition of the apparent horizon (31), a straightforward calculation gives

$$
\dot{\tilde{r}}_{A}=-\frac{1}{c^{2}} H \tilde{r}_{A}^{3}\left(\dot{H}-\frac{k}{a^{2}}\right) .
$$

Following [68], the work density $W$ and energy-supply vector $\Phi_{a}$ for our case can be calculated as follows:

$$
\begin{aligned}
W & =-\frac{1}{2} T^{a b} h_{a b}=-\frac{1}{2}(p-\rho), \\
\Phi_{a} & =T_{a}^{b} \partial_{b} \tilde{r}=-\frac{1}{2}(p+\rho) H \tilde{r} d t+\frac{1}{2}(p+\rho) a d r,
\end{aligned}
$$

with $T_{a b}$ the projection of the $(3+1)$-dimensional energy-momentum tensor $T_{\mu \nu}$ of matter in the normal direction of 2-sphere. Then the amount of energy crossing the apparent horizon during the time internal $d t$ is $[61]$

$$
d E=A \Phi=A(\rho+p) H \tilde{r}_{A} d t .
$$

Now, let us turn to the entropic force. Verlinde [46] made an interesting suggestion that gravity may be regarded as an entropic force caused by the changes in the information associated with the positions of material bodies. With this powerful assumption together with the holographic principle and the equipartition law of energy, Verlinde obtained the second law of Newtonian mechanics and Newtonian law of gravitation. The essence of the idea is that gravity is not fundamental but is an entropic force, which can be expressed in the following form

$$
F \Delta x=T \Delta S
$$


where $\Delta S$ denotes the small change of entropy $S$ of the gravitational system, $\Delta x$ is the displacement of a particle in the gravitational system, $T$ is the temperature of the system, and $F$ is named entropic force. In the original paper, the author suggested that the maximal storage space for information on the holographic screen, or total number of bits, is proportional to the area $A$ with the holographic principle is held, i.e.,

$$
N=\frac{A c^{3}}{G \hbar}
$$

where $A$ is area of the holographic screen. Then, with the equipartition law of energy $E=\frac{1}{2} N k_{B} T$ and $E=M c^{2}$ ( $M$ represents the mass that would emerge in the part of space enclosed by the holographic screen), one will recover the Newton's law of gravitation:

$$
F=G \frac{M m}{R^{2}}
$$

where the relations $F=m a$ ( $a$ is regarded as the acceleration corresponding to the Unruh temperature) and $A=4 \pi R^{2}$ are used.

Here, we propose that the total number of bits for the information is proportional to the entropy $S$ rather than the area $A$ in a gravity theory as did in [57]. Thus, for a general gravity theory, one could rewrite (13) as [70]

$$
N=\frac{4 S}{k_{B}} \text {. }
$$

Next, we will reproduce the Friedmann equation from this entropic force method. With (15), we obtain the total number of bits for the HL gravity

$$
N=\frac{A c^{3}}{G \hbar}+\frac{4 \pi}{\omega} \ln \frac{A c^{3}}{G \hbar}
$$

From the view of entropic force, the energy $E$ for a thermodynamic system can be reflected by the number of bits on the holographic screen through the equipartition law $E=\frac{1}{2} N k_{B} T$ with $T$ the temperature of the holographic screen. Here, we choose the apparent horizon to be the holographic screen. So the temperature $T$ is that for the apparent horizon. Then, we can get the change of the energy $d E$ from the equipartition law

$$
d E=\frac{1}{2} N k_{B} d T+\frac{1}{2} k_{B} T d N=\frac{8 G \hbar \pi+A \omega c^{3}-4 G \hbar \pi \ln \left(\frac{A c^{3}}{G \hbar}\right)}{4 A^{3 / 2} G \sqrt{\pi} \omega} c d A .
$$

Comparing (11) and (17), we reach

$$
\left[8 G \hbar \pi+A \omega c^{3}-4 G \hbar \pi \ln \left(\frac{A c^{3}}{G \hbar}\right)\right] d \tilde{r}_{A}=16 \pi^{2} c G \omega \tilde{r}_{A}^{5}(\rho+p) H d t .
$$


With the use of (8) and the continuity equation (44), we get

$$
\left[8 G \hbar \pi+A \omega c^{3}-4 G \hbar \pi \ln \left(\frac{A c^{3}}{G \hbar}\right)\right]\left(H-\frac{k}{a^{2}}\right) H=\frac{16 G \omega c^{3} \pi^{2} \tilde{r}_{A}^{2}}{3} \dot{\rho} .
$$

Using the relation

$$
2 H\left(\dot{H}-\frac{k}{a^{2}}\right)=\frac{d}{d t}\left(H^{2}+\frac{k}{a^{2}}\right)
$$

and integrating (19), we derive the following equation

$$
\frac{\hbar G}{4 \omega c^{5}}\left(H^{2}+\frac{k}{a^{2}}\right)^{2}\left[3-2 \ln \left(\frac{4 \pi c^{5}}{G \hbar\left(H^{2}+k / a^{2}\right)}\right)\right]+\left(H^{2}+\frac{k}{a^{2}}\right)=\frac{8 \pi G}{3} \rho,
$$

where the integration constant has also been absorbed into the energy density $\rho$. Eqs. (19) and (21) are the modified Friedmann equations from the entropic force. Comparing with the standard Friedmann equation (5), the modified one is in a complicated form. This means that in principle we can distinguish the HL gravity from the Einstein one by exploring its Friedmann equation. As we know, when the parameter $\omega$ of the HL gravity approaches infinite, the Einstein gravity will be recovered. Setting $\omega \rightarrow \infty$, we can see that the first term in (21) vanishes and the standard Friedmann equation will be obtained, which is exactly consistent with the result in [30].

In this letter, we obtain the modified Friedmann equation of FRW universe in the deformed HL gravity theory from the entropic force. In the deformed HL gravity, the entropy/area relation is generally considered to have a logarithmic term, i.e., $S=\frac{A}{4 G}+\frac{\pi}{\omega} \ln A$. The parameter $\omega$ can be regarded as a characteristic parameter in the deformed HL gravity and the entropy/area relation will recover to Bekenstein-Hawking entropy/area law when $\omega \rightarrow \infty$. To some extent, this logarithmic term represents a feature of black holes in the deformed HL gravity. Considering the apparent horizon of the universe is a holographic screen, we propose that the total number of bits for the information of the screen is $N=\frac{4 S}{k_{B}}$. Then the energy included in the screen can be described by the equipartition law of the energy. Thus we obtain the modified Friedmann equation, in which a square term of $\left(H^{2}+\frac{k}{a^{2}}\right)$ and a logarithmic term appeared, which provide a way to distinguish the HL gravity from the Einstein one. However, as the parameter $\omega \rightarrow \infty$, the modified equation will become the standard one. Our result may be suggested that gravity indeed has a thermodynamic origin for a non-Einstein gravity and it may be useful for further understand of the holographic properties and Friedmann equation for other gravity theories. 
Y.X. Liu would like to thank Dr. Li-Ming Cao for his helpful discussions. This work was supported by the Program for New Century Excellent Talents in University, the National Natural Science Foundation of China (No. 11075065), the Fundamental Research Funds for the Central Universities (No. lzujbky-2009-54, No. lzujbky-2009-163 and lzujbky-2009-122), and the Fundamental Research Fund for Physics and Mathematics of Lanzhou University (No. LZULL200912).

[1] E. M. Lifshitz, Zh. Eksp. Toer. Fiz. 11 (1941) 255.

[2] P. Hořava, Phys. Rev. D 79 (2009) 084008.

[3] P. Hořava, JHEP 0903 (2009) 020.

[4] P. Hořava, Phys. Rev. Lett. 102 (2009) 161301.

[5] H. Lu, J. Mei and C. N. Pope, Phys. Rev. Lett. 103 (2009) 091301.

[6] R. G. Cai, L. M. Cao and N. Ohta, Phys. Rev. D 80 (2009) 024003.

[7] R. G. Cai, Y. Liu and Y. W. Sun, JHEP 0906 (2009) 010.

[8] E. O. Colgain and H. Yavartanoo, JHEP 0908 (2009) 021.

[9] A. Kehagias and K. Sfetsos, Phys. Lett. B 678 (2009) 123.

[10] M. I. Park, JHEP 0909 (2009) 123.

[11] A. Ghodsi and E. Hatefi, Phys. Rev. D 81 (2010) 044016.

[12] H. W. Lee, Y. W. Kim and Y. S. Myung, Eur. Phys. J. C 68 (2010) 255.

[13] J. Z. Tang and B. Chen, Phys. Rev. D 81 (2010) 043515.

[14] M. R. Setare and D. Momeni, Int. J. Mod. Phys. D 19 (2010) 2079.

[15] E. Kiritsis, Phys. Rev. D 81 (2010) 044009.

[16] J. Z. Tang and B. Chen, Static Charged Black Hole Solutions in Hořava-Lifshitz Gravity, arXiv:0911.3849[hep-th].

[17] E. Ayon-Beato, A. Garbarz, G. Giribet and M. Hassaine, JHEP 04(2010)030.

[18] D. Capasso and A. P. Polychronakos, Phys. Rev. D 81 (2010) 084009.

[19] E. Kiritsis and G. Kofinas, JHEP 1001 (2010) 122.

[20] R. G. Cai, L. M. Cao and N. Ohta, Phys. Lett. B 679 (2009) 504; A.Wang and Y. Wu, J. Cosmol. Astropart. Phys. 0907 (2009) 012.

[21] Y. S. Myung, Phys. Lett. B 678 (2009) 127. 
[22] D. Y. Chen, H. T. Yang and X. T. Zu, Phys. Lett. B 681 (2009) 463.

[23] R. B. Mann, JHEP 0906 (2009) 075.

[24] Y. S. Myung, Phys. Lett. B 681 (2009) 81.

[25] Y. S. Myung, Phys. Lett. B 679 (2009) 491.

[26] S. B. Chen and J. L. Jing, Phys. Lett. B 687 (2010) 124.

[27] J. J. Peng and S. Q. Wu, Eur. Phys. J. C 66 (2010) 325.

[28] Y. S. Myung and Y. W. Kim, Eur. Phys. J. C 68 (2010) 265.

[29] R. G. Cai and N. Ohta, Phys. Rev. D 81 (2010) 084061.

[30] Q. J. Cao, Y. X. Chen and K. N. Shao, JCAP 05 (2010) 030.

[31] R. G. Cai and A. Z. Wang, Phys. Lett. B 686 (2010) 166.

[32] Y. S. Myung, Phys. Lett. B 685 (2010) 318.

[33] B. R. Majhi, Phys. Lett. B 686 (2010) 49.

[34] A. Z. Wang, D. Wands and R. Maartens, JCAP 03 (2010) 013.

[35] N. Varghese and V. C. Kuriakose, Stability of black holes in Horava gravity: Gravitational quasinormal modes, arXiv:0909.4944[gr-qc].

[36] C. K. Ding, S. B. Chen and J. L. Jing, Phys. Rev. D 81 (2010) 024028.

[37] Y. S. Myung, Phys. Lett. B 684 (2010) 158.

[38] M. J. Wang, J. L. Jing, C. K. Ding and S. B. Chen Phys. Rev. D 81 (2010) 083006.

[39] A. Castillo and A. Larranaga, Electron. J. Theor. Phys. 8 (2011) 1.

[40] M. R. Setare and M. Jamil, Int. J. Theor. Phys. 50 (2011) 511.

[41] X. Gao, Cosmological Perturbations and Non-Gaussianities in Hořava-Lifshitz Gravity, arXiv:0904.4187[hep-th].

[42] X. Gao, Y. Wang, R. Brandenberger and A. Riotto, Phys. Rev. D 81 (2010) 083508.

[43] X. Gao, Y. Wang, W. Xue and R. Brandenberger, Fluctuations in a Hořava-Lifshitz Bouncing Cosmology, arXiv:0911.3196 hep-th].

[44] S. Dutta and E. N. Saridakis, JCAP 1001 (2010) 013; G. Leon and E. N. Saridakis, JCAP 0911 (2009) 006; Y. F. Cai and E. N. Saridakis, JCAP 0910 (2009) 020.

[45] E. N. Saridakis, Eur. Phys. J. C 67 (2010) 229; C. Bogdanos and E. N. Saridakis, Class. Quant. Grav. 27 (2010) 075005.

[46] E. P. Verlinde, JHEP 1104 (2011) 029.

[47] A. D. Sakharov, Sov. Phys. Dokl. 12 (1968) 1040. 
[48] T. Padmanabhan, Mod. Phys. Lett. A 25 (2010) 1129; T. Padmanabhan, Class. Quant. Grav. 214485 (2004).

[49] F. W. Shu and Y. G. Gong, Int. J. Mod. Phys. D 20 (2011) 553.

[50] R. G. Cai, L. M. Cao and N. Ohta, Phys. Rev. D 81 (2010) 061501(R).

[51] L. Smolin, Newtonian gravity in loop quantum gravity, arXiv:1001.3668 gr-qc].

[52] J. Makela, Notes Concerning "On the Origin of Gravity and the Laws of Newton" by E. Verlinde (arXiv:1001.0785), arXiv:1001.3808[gr-qc].

[53] F. Caravelli and L. Modesto, Phys. Lett. B 702 (2011) 307.

[54] M. Li and Y. Wang, Phys. Lett. B 687 (2010) 243.

[55] C. J. Gao, Phys. Rev. D 81 (2010) 087306.

[56] T. Wang, Phys. Rev. D 81 (2010) 104045.

[57] Y. Zhang, Y. G. Gong and Z. H. Zhu, Int. J. Mod. Phys. D 20 (2011) 1505.

[58] F. Piazza, New views on the low-energy side of gravity, arXiv:0910.4677[gr-qc].

[59] H. Culetu, J. Korean Phys. Soc. 57 (2010) 17.

[60] Y. Wang, Towards a Holographic Description of Inflation and Generation of Fluctuations from Thermodynamics, arXiv:1001.4786[hep-th].

[61] R. G. Cai and S. P. Kim, JHEP 0502 (2005) 050.

[62] M. Akbar and R. G. Cai, Phys. Lett. B 635 (2006) 7.

[63] R. G. Cai, L. M. Cao and Y. P. Hu, JHEP 0808 (2008) 090.

[64] R. G. Cai, L. M. Cao and Y. P. Hu, Class. Quant. Grav. 26 (2009) 155018.

[65] R. G. Cai, Prog. Theor. Phys. Suppl. 172 (2008) 100.

[66] Y. G. Gong and A. Z. Wang, Phys. Rev. Lett. 99 (2007) 211301.

[67] T. ZhuJ. R. Ren and M. F. Li, Phys. Lett. B 674 (2009) 204; T. Zhu, J. R. Ren and D. Singleton, Int. J. Mod. Phys. D 19 (2010) 159.

[68] S. A. Hayward, Class. Quant. Grav. 15 (1998) 3147.

[69] T. Padmanabhan, Phys. Rev. D 81 (2010) 124040.

[70] In a general gravity theory, entropy $S$ is proportional to the total number of microstates $\Omega$. For $N \propto \ln \Omega$, so it is easy to obtain $N \propto S$. On the other hand, in the frame of Einstein gravity, we have $S \propto A$. Then it is straightforward to obtain $N \propto A$. This is also implied in the work [69]. 